\title{
Symbolic description of the polynomial roots and their numerical implementation - better than in Mathematica software?
}

\author{
Edyta Hetmaniok \\ Damian Słota \\ Mariusz Pleszczyński \\ Roman Wituła \\ and Michał Różański \\ Silesian University of Technology \\ Institute of Mathematics \\ Email: edyta.hetmaniok@polsl.pl \\ mariusz.pleszczynski@polsl.pl \\ roman.witula@polsl.pl \\ michal.rozanski@polsl.pl
}

\author{
Marcin Szczygieł \\ Silesian University of Technology \\ Institute of Mechatronics \\ Email: marcin.szczygiel@polsl.pl
}

\begin{abstract}
This paper is a continuation of the discussion undertaken in paper [31]. We present in the current paper the corrected, and also given in a slightly changed form, Vandermonde formulae for the roots of some quintic polynomials considered in J.P. Tignol's monograph [26]. The proofs of selected trigonometric identities from [31] are given and some new identities have been generated by the occasion, which also can be used for testing our Langrange algorithm for the case of cubic polynomials. Moreover, we present here the decomposition of polynomials belonging to some two-parameter family of polynomials related to the Chebyshev polynomials of the first kind.
\end{abstract}

Keywords - Lagrange algorithm, Chebyshev polynomials

\section{INTRODUCTION}

In paper [31] we have presented an algorithm (the so called Lagrange algorithm) for determining the complex roots of real polynomials which appeared to be more effective than the symbolic calculations proposed in Mathematica software. Many cubic polynomial, considering the polynomials used for testing our Lagrange algorithm, led us to some attractive trigonometric relations (see the appendix which is an important supplement of paper [31]). In the current paper we propose to repeat our previous considerations for the case of quintic polynomials and for the polynomials of higher degrees as well.

Let us also recall that the complex roots of quintic polynomials and the polynomials of higher degree, described with the aid of elliptic functions and hypergeometric functions, are well known (see [2], [12]). Certainly, the computational usefulness of such solutions is not good, it still remains in the sphere of future discoveries. At present, we have to limit the calculations, especially the symbolic calculations, just for the selected families of polynomials, for which the formulae describing roots are well known and are in the "numerically accepted" form.

\section{VANDERMONDE FORMULAE}

Next of the investigated quintic polynomials

$$
x^{5}-x^{4}-4 x^{3}+3 x^{2}+3 x-1=\prod_{k=1}^{5}\left(x+2 \cos \frac{2 k \pi}{11}\right)
$$

delivered to us many unexpected emotions. So in monograph [26] the following identity, based on the Lagrange resolvent method of solving the algebraic equations, is suggested

$$
x=\frac{1}{5}\left(1+\Delta^{\prime}+\Delta^{\prime \prime}+\Delta^{\prime \prime \prime}+\Delta^{I V}\right),
$$

where $x$ is one of the roots of this polynomial. However the description of expressions $\Delta^{\prime}, \Delta^{\prime \prime}, \Delta^{\prime \prime \prime}$ and $\Delta^{I V}$, given in [26], "seems to be" incorrect. It should be, and this is already our correction of these relations: $\Delta^{\prime}=\sqrt[5]{d_{1}}, \Delta^{\prime \prime}=\overline{\Delta^{\prime}}$, $\Delta^{\prime \prime \prime}=\sqrt[5]{d_{3}}, \Delta^{I V}=\overline{\Delta^{\prime \prime \prime}}$, more precisely, $\Delta^{\prime}$ and $\Delta^{\prime \prime \prime}$ are any values of the respective complex roots of degree five taken from numbers (respectively):

$$
\begin{aligned}
& d_{1}=\frac{11}{4}(89+25 \sqrt{5}-5 i \sqrt{5+2 \sqrt{5}}+45 i \sqrt{5-2 \sqrt{5}}), \\
& d_{3}=\frac{11}{4}(89-25 \sqrt{5}-45 i \sqrt{5+2 \sqrt{5}}-5 i \sqrt{5-2 \sqrt{5}}) .
\end{aligned}
$$

Identity (1) takes then the following trigonometric form ${ }^{1}$ (the calculations were executed by hand with the intensive support

\footnotetext{
${ }^{1}$ We used in calculations the following identity$$
d \sqrt{a+b \sqrt{a}}+\sqrt{a-b \sqrt{a}}=\sqrt{\left(d^{2}+1\right) a+\left(b\left(d^{2}-1\right)+2 c d\right) \sqrt{a}},
$$

where $a=b^{2}+c^{2}, b, c \in \mathbb{C}$ (the complex cases need some additional verification).
} 
of Mathematica software):

$$
\begin{aligned}
-2 \cos \frac{2 k \pi}{11} & =\frac{1}{5}\left(1+2 \sqrt{11} \cos \left(\frac{1}{5}\left(t_{i}+s_{i} \arctan \frac{\Im d_{1}}{\Re d_{1}}\right)\right)\right. \\
& \left.+\sqrt{11} \cos \left(\frac{1}{5}\left(t_{j}-r_{j} \arctan \frac{\Im d_{3}}{\Re d_{3}}\right)\right)\right)
\end{aligned}
$$

and hence, after simple algebraic transformations, we get

$$
\begin{aligned}
-5 \cos \frac{2 k \pi}{11}-\frac{1}{2} & =\sqrt{11}\left(\cos \left(\frac{1}{5}\left(t_{i}+s_{i} \arctan \frac{p}{q}\right)\right)\right. \\
& \left.+\cos \left(\frac{1}{5}\left(t_{j}+r_{j} \arctan \frac{p q}{109}\right)\right)\right),
\end{aligned}
$$

where $p=5 \sqrt[4]{5}, q=\sqrt{877 \sqrt{5}+1958}, s_{i}, r_{i}$ are the signs and $t_{n}:=2(n-1) \pi$ for every $n=1,2, \ldots, 5$. By using the Mathematica software we have found the following sequences of the values of indices $k, i, j$ and the signs $s_{i}, r_{j}$ for which both the above identities hold true:

\begin{tabular}{ccccc||ccccc}
$k$ & $i$ & $j$ & $s_{i}$ & $r_{j}$ & $k$ & $i$ & $j$ & $s_{i}$ & $r_{j}$ \\
\hline 1 & 3 & 3 & -1 & -1 & 3 & 1 & 4 & +1 & -1 \\
1 & 3 & 4 & -1 & +1 & 3 & 1 & 4 & -1 & -1 \\
1 & 4 & 3 & +1 & -1 & 4 & 2 & 2 & +1 & -1 \\
1 & 4 & 4 & +1 & +1 & 4 & 2 & 5 & +1 & +1 \\
2 & 3 & 2 & +1 & +1 & 4 & 5 & 2 & -1 & -1 \\
2 & 3 & 5 & +1 & -1 & 4 & 5 & 5 & -1 & +1 \\
2 & 4 & 2 & -1 & +1 & 5 & 2 & 1 & -1 & -1 \\
2 & 4 & 5 & -1 & -1 & 5 & 2 & 1 & -1 & +1 \\
3 & 1 & 3 & +1 & +1 & 5 & 5 & 1 & +1 & -1 \\
3 & 1 & 3 & -1 & +1 & 5 & 5 & 1 & +1 & +1 \\
\hline
\end{tabular}

III. Quintic Polynomials

In this section we discuss the monic irreducible quintic polynomials $f \in \mathbb{Q}[x]$. If the equation $f(x)=0$ is solvable by radicals, the quintic polynomial $f(x)$ is said to be solvable. First let us consider the quintic polynomial in the reduced form

$$
x^{5}+a_{2} x^{2}+a_{1} x+a_{0}=0,
$$

called the principal quintic form, and

$$
x^{5}+b_{1} x+b_{0}=0,
$$

called the Bring-Jerrard quintic form (see [1]).

It is known that if $f(x)=x^{5}+a x^{i}+b \in \mathbb{Q}^{*}[x], i=1,2$, where $\mathbb{Q}^{*}:=\mathbb{Q} \backslash\{0\}$, and $\operatorname{disc} f(x)>0^{2}$, then $f(x)$ has exactly one real root [24].

B.K. Spearman and K.S. Williams proved in [23] the following result.

Theorem 1. Let $a, b \in \mathbb{Q}^{*}$ be such that the quintic trinomial $f(x)=x^{5}+a x+b$ is irreducible. Then $f(x)$ is solvable if and only if there exist the rational numbers $\varepsilon \in\{-1,1\}, c \geq 0$ and $\alpha \neq 0$ such that

$$
a=\frac{5 \alpha^{4}(3-4 \varepsilon c)}{c^{2}+1}, \quad b=\frac{-4 \alpha^{5}(11 \varepsilon+2 c)}{c^{2}+1} .
$$

Moreover, if we set

$$
D=c^{2}+1
$$

${ }^{2}$ The discriminant of $f$.
$\begin{cases}v_{1}=\sqrt{D}+\sqrt{D-\varepsilon \sqrt{D}}, & v_{2}=-\sqrt{D}-\sqrt{D+\varepsilon \sqrt{D}}, \\ v_{3}=-\sqrt{D}+\sqrt{D+\varepsilon \sqrt{D}}, & v_{4}=\sqrt{D}-\sqrt{D-\varepsilon \sqrt{D}},\end{cases}$

$u_{1}=\sqrt[5]{\frac{v_{1}^{2} v_{3}}{D^{2}}}, u_{2}=\sqrt[5]{\frac{v_{3}^{2} v_{4}}{D^{2}}}, u_{3}=\sqrt[5]{\frac{v_{2}^{2} v_{1}}{D^{2}}}, u_{4}=\sqrt[5]{\frac{v_{4}^{2} v_{2}}{D^{2}}}$,

and $w=\exp (2 \pi i / 5)$, then the roots of $x^{5}+a x+b$ are equal to $x_{0}, x_{1}, x_{2}, x_{3}, x_{4}$, where

$$
\begin{aligned}
& \frac{x_{i}}{\alpha}=\sum_{k=1}^{4} w^{k i} u_{k} \\
& =w^{i} u_{1}+\frac{w^{2 i} u_{1}^{2} \sqrt{D} \sqrt[5]{\varepsilon}}{v_{1}}+\frac{w^{3 i} u_{1}^{3} D}{v_{1} v_{3}}-\frac{w^{4 i} u_{1}^{4} \sqrt{D^{3}}}{v_{1}^{2} v_{3}} \sqrt[5]{\frac{\varepsilon}{D}}
\end{aligned}
$$

for every $i=0,1,2,3,4$.

For illustrating the application of the formulae from the above theorem let us find the complex roots of polynomial

$$
x^{5}+11 x+44
$$

which we get for parameters $\varepsilon=1, \alpha=-1, c=2 / 11$. By using the above formulae, supported by Mathematica software, we derive the following formulae

$$
\begin{aligned}
x_{0} & =\lambda\left(\xi_{1}-\xi_{2}-\xi_{3}-\xi_{4}\right) \approx-1.87775, \\
x_{1} & =-\lambda\left(e^{\frac{3 \pi i}{5}} \xi_{1}+e^{\frac{2 \pi i}{5}} \xi_{2}+e^{\frac{4 \pi i}{5}} \xi_{3}-e^{\frac{\pi i}{5}} \xi_{4}\right) \\
& \approx 1.80012-1.44746 i, \\
x_{2} & =-\lambda\left(e^{\frac{\pi i}{5}} \xi_{1}+e^{\frac{4 \pi i}{5}} \xi_{2}-e^{\frac{3 \pi i}{5}} \xi_{3}+e^{\frac{2 \pi i}{5}} \xi_{4}\right) \\
& \approx-0.861241-1.9105 i, \\
x_{3} & =\overline{x_{2}}, \quad x_{4}=\overline{x_{1}},
\end{aligned}
$$

where $\lambda=-\sqrt[5]{11 / 5^{4}}$ and

$$
\begin{gathered}
\xi_{k}:=\left((-1)^{k+1}(50 \sqrt{5}-75)+\sqrt{18125-6169 \sqrt{5}}\right)^{1 / 5}, \\
\xi_{l}:=\left(50 \sqrt{5}+75+(-1)^{l} \sqrt{18125+6169 \sqrt{5}}\right)^{1 / 5},
\end{gathered}
$$

for $k=1,2$ and $l=3,4$.

J.A. Johnstone and B.K. Spearman in paper [11] proved the following result.

Theorem 2. Let $f(x)=x^{5}+x-a$, where $a \in \mathbb{Z}$. Then $f(x)$ is not solvable by radicals unless $a=r^{5}+r$ for some integer $r$, or $a= \pm 1, \pm 6$. The last case was discussed and proved earlier by S. Rabinowitz [21].

B.K. Spearman and K.S. Williams in paper [24] proved also the completely unexpected result, given below.

Theorem 3. There exist only five essentially different, irreducible, solvable, quintic trinomials $x^{5}+a x^{2}+b, a, b \in \mathbb{Q}^{*}$, namely: $x^{5}+5 x^{2}+3, x^{5}+5 x^{2}-15, x^{5}+25 x^{2}+300$, $x^{5}+100 x^{2}+1000$ and $x^{5}+250 x^{2}+625$. 


\section{APPENDIX}

In paper [31] we have presented the following formulae

$$
\begin{aligned}
& \left(z-2 \sin \frac{4 \pi}{13}\right)\left(z-2 \sin \frac{10 \pi}{13}\right)\left(z-2 \sin \frac{12 \pi}{13}\right) \\
& =z^{3}-\sqrt{\frac{13+3 \sqrt{13}}{2}} z^{2}+\sqrt{13} z-\sqrt{\frac{13-3 \sqrt{13}}{2}} \\
& \left(z-2 \sin \frac{2 \pi}{13}\right)\left(z-2 \sin \frac{6 \pi}{13}\right)\left(z+2 \sin \frac{8 \pi}{13}\right) \\
& =z^{3}-\sqrt{\frac{13-3 \sqrt{13}}{2}} z^{2}-\sqrt{13} z+\sqrt{\frac{13+3 \sqrt{13}}{2}}
\end{aligned}
$$

with the suggestion that they can be derived on the basis of formulae given below

$$
\begin{aligned}
& z^{3}+\frac{1-\sqrt{13}}{2} z^{2}-z+\frac{\sqrt{13}+3}{2} \\
& =\left(z-2 \cos \frac{2 \pi}{13}\right)\left(z-2 \cos \frac{6 \pi}{13}\right)\left(z-2 \cos \frac{8 \pi}{13}\right), \\
& z^{3}+\frac{1+\sqrt{13}}{2} z^{2}-z-\frac{\sqrt{13}+3}{2} \\
& =\left(z-2 \cos \frac{4 \pi}{13}\right)\left(z-2 \cos \frac{10 \pi}{13}\right)\left(z-2 \cos \frac{12 \pi}{13}\right) .
\end{aligned}
$$

To explain it better we decided to "reveal" here the details of the proof. Thus, by proving decomposition (3) and (4) we used the following relations

$$
\begin{aligned}
& 8 \sin \frac{4 \pi}{13} \sin \frac{10 \pi}{13} \sin \frac{12 \pi}{13}=i\left(e^{i \frac{4 \pi}{13}}-e^{-i \frac{4 \pi}{13}}\right) \\
& \times\left(e^{i \frac{10 \pi}{13}}-e^{-i \frac{10 \pi}{13}}\right)\left(e^{i \frac{12 \pi}{13}}-e^{-i \frac{12 \pi}{13}}\right) \\
& =2 \sin \frac{2 \pi}{13}+2 \sin \frac{6 \pi}{13}-2 \sin \frac{8 \pi}{13}, \\
& \left(2 \sin \frac{4 \pi}{13}+2 \sin \frac{10 \pi}{13}+2 \sin \frac{12 \pi}{13}\right)^{2} \\
& =6+2\left(\cos \frac{2 \pi}{13}+\cos \frac{6 \pi}{13}+\cos \frac{8 \pi}{13}\right) \\
& -4\left(\cos \frac{4 \pi}{13}+\cos \frac{10 \pi}{13}+\cos \frac{12 \pi}{13}\right) \underset{(6)}{\stackrel{(5)}{=} \frac{13+3 \sqrt{13}}{2}}, \\
& 4\left(\sin \frac{4 \pi}{13} \sin \frac{10 \pi}{13}+\sin \frac{4 \pi}{13} \sin \frac{12 \pi}{13}+\sin \frac{10 \pi}{13} \sin \frac{12 \pi}{13}\right)= \\
& =2\left(\cos \frac{2 \pi}{13}+\cos \frac{6 \pi}{13}+\cos \frac{8 \pi}{13}\right) \\
& -2\left(\cos \frac{4 \pi}{13}+\cos \frac{10 \pi}{13}+\cos \frac{12 \pi}{13}\right) \underset{(6)}{\stackrel{(5)}{=}} \sqrt{13}, \\
& 8 \sin \frac{2 \pi}{13} \sin \frac{6 \pi}{13} \sin \frac{8 \pi}{13} \\
& =i\left(e^{i \frac{2 \pi}{13}}-e^{-i \frac{2 \pi}{13}}\right)\left(e^{i \frac{6 \pi}{13}}-e^{-i \frac{6 \pi}{13}}\right)\left(e^{i \frac{8 \pi}{13}}-e^{-i \frac{8 \pi}{13}}\right) \\
& =2 \sin \frac{4 \pi}{13}+2 \sin \frac{10 \pi}{13}+2 \sin \frac{12 \pi}{13},
\end{aligned}
$$

$$
\begin{aligned}
& \left(2 \sin \frac{2 \pi}{13}+2 \sin \frac{6 \pi}{13}-2 \sin \frac{8 \pi}{13}\right)^{2} \\
& =6+2\left(\cos \frac{4 \pi}{13}+\cos \frac{10 \pi}{13}+\cos \frac{12 \pi}{13}\right) \\
& -4\left(\cos \frac{2 \pi}{13}+\cos \frac{6 \pi}{13}-\cos \frac{8 \pi}{13}\right) \frac{(5)}{=} \frac{13-3 \sqrt{13}}{2} \\
& 4\left(\sin \frac{2 \pi}{13} \sin \frac{6 \pi}{13}-\sin \frac{2 \pi}{13} \sin \frac{8 \pi}{13}-\sin \frac{6 \pi}{13} \sin \frac{8 \pi}{13}\right) \\
& =2\left(\cos \frac{4 \pi}{13}+\cos \frac{10 \pi}{13}+\cos \frac{12 \pi}{13}\right) \\
& -2\left(\cos \frac{2 \pi}{13}+\cos \frac{6 \pi}{13}+\cos \frac{8 \pi}{13}\right) \frac{(5)}{(6)}-\sqrt{13}
\end{aligned}
$$

In order to complete the collection of formulae (3)-(6) we give additionally the decompositions connected with the values of tangent and cotangent functions of the respective ternary sets of arguments $\left\{\frac{2 \pi}{13}, \frac{6 \pi}{13}, \frac{8 \pi}{13}\right\}$ and $\left\{\frac{4 \pi}{13}, \frac{10 \pi}{13}, \frac{12 \pi}{13}\right\}$. They are of the following form

$$
\begin{aligned}
& \left(x-\tan \frac{4 \pi}{13}\right)\left(x-\tan \frac{10 \pi}{13}\right)\left(x-\tan \frac{12 \pi}{13}\right) \\
& =x^{3}-\sqrt{65-18 \sqrt{13}} x^{2}+(13-4 \sqrt{13}) x-\sqrt{65-18 \sqrt{13}},
\end{aligned}
$$

since from formulae (3)-(6) we easily obtain the equalities

$$
\begin{aligned}
& \tan \frac{4 \pi}{13} \tan \frac{10 \pi}{13} \tan \frac{12 \pi}{13}=\frac{2 \sin \frac{4 \pi}{13} 2 \sin \frac{10 \pi}{13} 2 \sin \frac{12 \pi}{13}}{2 \cos \frac{4 \pi}{13} 2 \cos \frac{10 \pi}{13} 2 \cos \frac{12 \pi}{13}} \\
&= \frac{\sqrt{\frac{13-3 \sqrt{13}}{2}}}{\frac{\sqrt{13}+3}{2}}=\frac{\sqrt{13}-3}{4} \sqrt{26-6 \sqrt{13}}=\sqrt{65-18 \sqrt{13}}, \\
& \tan \frac{4 \pi}{13}+\tan \frac{10 \pi}{13}+\tan \frac{12 \pi}{13}=\tan \frac{4 \pi}{13} \tan \frac{10 \pi}{13} \tan \frac{12 \pi}{13},{ }^{3}
\end{aligned}
$$

${ }^{3}$ The following identity holds

$$
\tan x+\tan y+\tan (k \pi-x-y)=\tan x \tan y \tan (k \pi-x-y)
$$

for the respective values of $x, y \in \mathbb{R}$ and $k \in \mathbb{Z}$, which in turn implies the identity

$$
\cot x \cot y+\cot x \cot (k \pi-x-y)+\cot y \cot (k \pi-x-y)=1 .
$$

Moreover, if $x_{1}+x_{2}+x_{3}=2 \pi, x_{1}, x_{2}, x_{3} \in \mathbb{R} \backslash \pi \mathbb{Z}$, then the following identities hold

$$
\begin{aligned}
& -2\left(\cot x_{1}+\cot x_{2}+\cot x_{3}\right) \sin x_{1} \sin x_{2} \sin x_{3} \\
& \quad=\sin ^{2} x_{1}+\sin ^{2} x_{2}+\sin ^{2} x_{3}=\left(\sin x_{1}+\sin x_{2}+\sin x_{3}\right)^{2} \\
& \quad-2 \sin x_{1} \sin x_{2}-2 \sin x_{1} \sin x_{3}-2 \sin x_{2} \sin x_{3}
\end{aligned}
$$

Proof. We have

$$
\begin{aligned}
& \cot x_{1}+\cot x_{2}=-\frac{\sin ^{2} x_{3}}{\sin x_{1} \sin x_{2} \sin x_{3}}, \\
& \cot x_{1}+\cot x_{3}=-\frac{\sin ^{2} x_{2}}{\sin x_{1} \sin x_{2} \sin x_{3}}, \\
& \cot x_{2}+\cot x_{3}=-\frac{\sin ^{2} x_{1}}{\sin x_{1} \sin x_{2} \sin x_{3}},
\end{aligned}
$$

from which, by summing respectively by sides, we obtain the expected equality. 
the last formula implies the following one

$$
\begin{gathered}
\cot \frac{4 \pi}{13}+\cot \frac{10 \pi}{13}+\cot \frac{12 \pi}{13}=\frac{\frac{1}{4} \frac{13+3 \sqrt{13}}{2}-\frac{\sqrt{13}}{2}}{-\frac{1}{4} \sqrt{\frac{13-3 \sqrt{13}}{2}}} \\
=-\frac{\sqrt{2}}{4} \sqrt{13+3 \sqrt{13}}(\sqrt{13}-1)=-\frac{\sqrt{2}}{2} \sqrt{26+4 \sqrt{13}} \\
\tan \frac{4 \pi}{13} \tan \frac{10 \pi}{13}+\tan \frac{4 \pi}{13} \tan \frac{12 \pi}{13}+\tan \frac{10 \pi}{13} \tan \frac{12 \pi}{13} \\
=\tan \frac{4 \pi}{13} \tan \frac{10 \pi}{13} \tan \frac{12 \pi}{13}\left(\cot \frac{4 \pi}{13}+\cot \frac{10 \pi}{13}+\cot \frac{12 \pi}{13}\right) \\
=\sqrt{65-18 \sqrt{13}}\left(-\frac{\sqrt{2}}{2} \sqrt{26+4 \sqrt{13}}\right) \\
=-\sqrt{(65-18 \sqrt{13})(13+2 \sqrt{13})}=13-4 \sqrt{13} .
\end{gathered}
$$

The next formulae, derived by us on the way of algebraic tests of the Lagrange algorithm, are as follows (we present them without the proofs with respect to the length of this paper):

$$
\begin{aligned}
& \left(x-\cot \frac{4 \pi}{13}\right)\left(x-\cot \frac{10 \pi}{13}\right)\left(x \cot \frac{12 \pi}{13}\right) \\
& =x^{3}+\frac{\sqrt{2}}{2} \sqrt{26+4 \sqrt{13}} x^{2}+x-\sqrt{\frac{1}{13}(65+18 \sqrt{13})}, \\
& \left(x-\tan \frac{2 \pi}{13}\right)\left(x-\tan \frac{6 \pi}{13}\right)\left(x+\tan \frac{8 \pi}{13}\right)=x^{3}- \\
& \sqrt{65-18 \sqrt{13}} x^{2}+(13+4 \sqrt{13}) x-\sqrt{65-18 \sqrt{13}}, \\
& \left(x-\cot \frac{2 \pi}{13}\right)\left(x-\cot \frac{6 \pi}{13}\right)\left(x+\cot \frac{8 \pi}{13}\right) \\
& =x^{3}-\sqrt{13-2 \sqrt{13}} x^{2}+x-\sqrt{\frac{1}{13}(65-18 \sqrt{13})}, \\
& \left(x-2 \cos \frac{2 \pi}{21}\right)\left(x-2 \cos \frac{8 \pi}{21}\right)\left(x-2 \cos \frac{32 \pi}{21}\right) \\
& =\left(x-2 \cos \frac{2 \pi}{21}\right)\left(x-2 \cos \frac{8 \pi}{21}\right)\left(x+2 \cos \frac{11 \pi}{21}\right) \\
& =x^{3}-\frac{1+\sqrt{21}}{2} x^{2}-\frac{1-\sqrt{21}}{2} x-\frac{5-\sqrt{21}}{2} \text {, } \\
& \left(x-2 \cos \frac{4 \pi}{21}\right)\left(x-2 \cos \frac{16 \pi}{21}\right)\left(x-2 \cos \frac{64 \pi}{21}\right) \\
& =\left(x-2 \cos \frac{4 \pi}{21}\right)\left(x+2 \cos \frac{5 \pi}{21}\right)\left(x+2 \cos \frac{\pi}{21}\right) \\
& =x^{3}-\frac{1-\sqrt{21}}{2} x^{2}-\frac{1+\sqrt{21}}{2} x-\frac{5+\sqrt{21}}{2} \text {, }
\end{aligned}
$$

since $\cos \frac{16 \pi}{21}=-\cos \frac{5 \pi}{21}, \cos \frac{32 \pi}{21}=\cos \frac{10 \pi}{21}=-\cos \frac{11 \pi}{21}$ and $\cos \frac{64 \pi}{21}=-\cos \frac{\pi}{21}=\cos \frac{20 \pi}{21}$. Finally the last formula is of the form

$$
\begin{gathered}
\prod_{k=1}^{6}\left(x-2 \cos \frac{2^{k} \pi}{21}\right)= \\
=\left(x^{3}-\frac{1+\sqrt{21}}{2} x^{2}-\frac{1-\sqrt{21}}{2} x-\frac{5-\sqrt{21}}{2}\right) \\
\times\left(x^{3}-\frac{1-\sqrt{21}}{2} x^{2}-\frac{1+\sqrt{21}}{2} x-\frac{5+\sqrt{21}}{2}\right) \\
=x^{6}-x^{5}-6 x^{4}+6 x^{3}+8 x^{2}-8 x+1 .
\end{gathered}
$$

Furthermore we have (see [29]):

$$
\begin{aligned}
& \left(x-2 \sin \frac{2 \pi}{21}\right)\left(x-2 \sin \frac{8 \pi}{21}\right)\left(x-2 \sin \frac{32 \pi}{21}\right) \\
& =\left(x-2 \sin \frac{2 \pi}{21}\right)\left(x-2 \sin \frac{8 \pi}{21}\right)\left(x+2 \sin \frac{10 \pi}{21}\right) \\
& =x^{3}+\frac{\sqrt{3}-\sqrt{7}}{2} x^{2}-\frac{3+\sqrt{21}}{2} x+\frac{\sqrt{3}+\sqrt{7}}{2}, \\
& \left(x-2 \sin \frac{4 \pi}{21}\right)\left(x-2 \sin \frac{16 \pi}{21}\right)\left(x-2 \sin \frac{64 \pi}{21}\right) \\
& =\left(x-2 \sin \frac{4 \pi}{21}\right)\left(x-2 \sin \frac{5 \pi}{21}\right)\left(x+2 \sin \frac{\pi}{21}\right) \\
& =x^{3}-\frac{\sqrt{3}+\sqrt{7}}{2} x^{2}+\frac{\sqrt{21}-3}{2} x+\frac{\sqrt{7}-\sqrt{3}}{2},
\end{aligned}
$$

since $\sin \frac{16 \pi}{21}=\sin \frac{5 \pi}{21}, \sin \frac{32 \pi}{21}=-\sin \frac{10 \pi}{21}=-\sin \frac{11 \pi}{21}$ and $\sin \frac{64 \pi}{21}=-\sin \frac{\pi}{21}=-\sin \frac{20 \pi}{21}$, which implies

$$
\begin{aligned}
& \prod_{k=1}^{6}\left(x-2 \sin \frac{2^{k} \pi}{21}\right) \\
& \quad=x^{6}-\sqrt{7} x^{5}-2 x^{4}+4 \sqrt{7} x^{3}-8 x^{2}+1 .
\end{aligned}
$$

\section{SPECIAL FAMILIES OF POLYNOMIALS}

There exist many special families of polynomials with the well known sets of roots. However there is no any common platform connecting these families and this is, to be honest, a great challenge of our undertaking. Considering the special families of polynomials, discovered by us, we want to emphasize these ones, the origins of which can be found in Cardano's formulae (see for example [28]). So if $\Omega_{n}(x)$ denote the so called $n$-th Chebyshev polynomial defined by the relation

$$
\Omega_{n}(x):=2 T_{n}\left(\frac{x}{2}\right),
$$

where $T_{n}(\cos \theta)=\cos n \theta$ is the $n$-th Chebyshev polynomial of the first kind, then the following decompositions hold

$$
\begin{gathered}
(\sqrt{p})^{n} \Omega_{n}\left(\frac{x}{\sqrt{p}}\right)-q=\prod_{k=0}^{n-1}\left(x-a \zeta^{2 k}-b \zeta^{-2 k}\right), \\
(-\sqrt{-p})^{n} \Omega_{n}\left(\frac{i x}{\sqrt{p}}\right)+q=\prod_{k=0}^{n-1}\left(x-a \zeta^{2 k+1}+b \zeta^{-2 k-1}\right),
\end{gathered}
$$


where $\zeta=\exp (i \pi / n), a$ and $b$ are any values from among the $n$ complex values of the following roots (respectively):

$$
\sqrt[n]{\frac{1}{2}\left(q+\sqrt{q^{2}-4 p^{n}}\right)}, \quad \sqrt[n]{\frac{1}{2}\left(q-\sqrt{q^{2}-4 p^{n}}\right)} .
$$

These formulae lead to many special cases, they generate many trigonometric identities. However the disadvantage of these identities is their dependence only on two complex parameters $p$ and $q$, no matter what is the value of the discussed polynomial degree. We also observe some calculating difficulties - however just on the same kind as in case of the classical Cardano's formulae.

\section{CONCLUSIONS}

Our attempt of making the algorithms, serving for symbolic determination of the complex roots of some real polynomials, more effective brought us many practical advantages. We have obtained the algorithms behaving better, at least in the cases emphasized by us, in comparison with, for example, the procedures used in Mathematica software. By the way we have generated many original identities and we have discovered new relations. We have also learned to avoid verious traps appearing in the course of using the algebra of complex numbers. Considering the future plans we intend still to seek the families of polynomials with the known description of roots. Also the form of realizing our mega-algorithm, which should end our future investigations, still remains questionable.

\section{REFERENCES}

[1] V.S. Adamchik and D.J. Jeffrey, "Polynomial Transformations of Tschirnhaus, Bring and Jerrard", ACM SIGSAM Bulletin, vol. 37, no 3, 2003, pp. 90-94.

[2] G. Belardinelli, Fonctions hypergéométriques de plusieurs variables et résolution analytique des équations algébriques générales, GauthierVillars, Paris 1960.

[3] B.C. Berndt, B.K. Spearman and K.S. Williams, "Commentary on an unpublished lecture by G.N. Watson on solving the quintic", Math. Intelligencer, vol. 24, 2002, pp. 15-33.

[4] D.S. Dummit, "Solving solvable quintics", Math. Comp., vol. 57, 1991, pp. $387-401$.

[5] M. Elia and P. Filipponi, "Equations of the Bring-Jerrard form, the golden section, and square fibonacci numbers", The Fibonacci Quarterly, vol. 36 no. 3 , 1998 , pp. 282-286.

[6] H. Funato and A. Kawamura, "Control of variable active-passive reactance (VAPAR) and negative inductance", PESC '94 Record., 25th Аnnual IEEE, pp. 189-196 vol.1, ISBN 0-7803-1859-5, DOI 10.1109/PESC.1994.349731.

[7] J.C. Glashan, "Notes on the quintic", Amer. J. Math., vol. 7, no. 2, 1885, pp. $178-179$.

[8] L. Guishu, D. Huaying, W. Tao and C. Xiang, "Generalized Kuroda's identity and its applications in nonuniform transmission lines", Proceedings. 2003 6th ISAPE, pp. 839-842, ISBN 0-7803-7831-8, DOI 10.1109/ISAPE.2003.1276817.

[9] T.R. Hagedorn, "General formulas for solving solvable sextic equations", J. Algebra, vol. 233, no. 2, 2000, pp. 704-751.

[10] E. Hetmaniok, P. Lorenc, M. Pleszczyński and R. Wituła, "Iterated integrals of polynomials", Appl. Math. Comput., vol. 249, 2014, pp. $389-398$.

[11] J.A. Johnstone and B.K. Spearman, "On a sequence of nonsolvable quintic polynomials", J. Integer Seq., vol. 12, no. 2, 2009, Art. ID 09.2.8.

[12] F. Klein, Vorlesungen Über das Ikosaeder und die Auflösung der Gleichungen vom Füntten Grade, Leipzig 1884 (we used the Russian translation: Nauka Press, Moscow 1989).
[13] S. Landau, " $\sqrt{2}+\sqrt{3}$ : four different views", The Math. Intelligencer, vol. 20 , no. 4 , 1998 , pp. $55-60$.

[14] S. Landau and G.L. Miller, "Solvability by radicals is in polynomial time", J. Comp. System Sciences, vol. 30, 1985, pp. 179-208.

[15] C.A. Leal-Sevillano, J.R. Montejo-Garai, J.A. Ruiz-Cruz and J.M. Rebollar, "Wideband Equivalent Circuit for Multi-Aperture Multi-Resonant Waveguide Irises", IEEE TMTT, vol. 64, iss. 3, pp.724-732, ISSN 00189480, DOI 10.1109/TMTT.2016.2520462.

[16] E. Mc Clintock, "Further researches in the theory of quintic equations", Amer. J. Math., vol. 20, no. 2, 1898, pp. 157-192.

[17] A. Mostowski and M. Stark, Elements of the higher algebra, PWN, Warszawa 1972 (in Polish).

[18] L.J. Okunev, Ring of polynomials and field of rational functions, in Mathematics, its subject, methods and significance, Part II, Academy of Science Press, Moscow 1951 (in Russian).

[19] F.W.I. Olver, D.W. Lozier, R.F. Boisvert and C.W. Clark, NIST Handbook of Mathematical Functions, Cambridge Univ. Press, 2010.

[20] A.P. Prudnikov, A.J. Bryczkov and O.I. Mariczev, Integrals and series, Elementary Functions, Nauka, Moscow 1981 (in Russian).

[21] S. Rabinowitz, "The factorization of $x^{5} \pm x+n$ ", Math. Magazine, vol. 61, 1988, pp. 191-193.

[22] B. Resmana, R.P. Astuti and A. Kurniawan, Wiener filter-based channel predictor performance improvement using polinomial extrapolation, 3rd ICICI-BME, Bandung 2013, pp.184-189, ISBN 978-1-4799-1649-8, DOI 10.1109/ICICI-BME.2013.6698489.

[23] B.K. Spearman and K.S. Williams, "Characterization of solvable quintics $x^{5}+a x+b "$, Amer. Math. Mon., vol. 101, no 10, 1994, pp. 986-992.

[24] B.K. Spearman and K.S. Williams, "On solvable quintics $x^{5}+a x+b$ and $x^{5}+a x^{2}+b ", \quad$ Rocky Mountain J. Math., vol. 26, 1996, pp. 753-772.

[25] B.K. Spearman and K.S. Williams, "The factorization of $x^{5} \pm x^{a}+n$ ", The Fibonacci Quarterly, vol. 36, 1998, pp. 158-170.

[26] J.-P. Tignol, Galois' Theory of Algebraic Equations, World Scientific, New Jersey 2001.

[27] R. Wituła, E. Hetmaniok, D. Słota and N. Gawrońska, "Sums of the rational powers of roots of the polynomials", International Journal of Pure and Applied Mathematics, vol. 85, no. 1, 2013, pp. 179-191.

[28] R. Wituła and D. Słota, "Cardano's formula, square roots, Chebyshev polynomials and radicals", J. Math. Anal. Appl., vol. 363, 2010, pp. 639647.

[29] R. Wituła and D. Słota, "Fixed and periodic points of polynomials generated by minimal polynomials of $2 \cos (2 \pi / n)$ ", Int. J. Bifur. Chaos, vol. 19 , no. 9,2009 , pp. 1-12.

[30] R. Wituła and D. Słota, "On modified Chebyshev polynomials", J. Math. Anal. Appl., vol. 324, 2006, pp. 321-343. Corrigendum to "On modified Chebyshev polynomials", J. Math. Anal. Appl., vol. 336, 2007, p. 750.

[31] A. Wróbel, E. Hetmaniok, M. Pleszczyński and R. Wituła, "On an improvement of the numerical application for Cardano's formula in Mathematica software", Proc. Inter. Symp. Young Scien. Techn., Eng. Math. SYSTEM 2015, Catania, Italy, 2015, pp. 27-29, online: http://ceurws.org/Vol-1543/p10.pdf. 